\title{
Variations of the Cervical Internal Carotid Artery
}

\author{
Rajendra B. Metgudmath · Anjali R. Metgudmath • \\ Vinita V. Metgudmath • Vivek Jainkeri
}

Received: 28 September 2011/ Accepted: 24 November 2011/Published online: 6 January 2012

(C) Association of Otolaryngologists of India 2012

\begin{abstract}
The objective of this study was to report the variations of the cervical internal carotid artery, as encountered during neck dissection for head and neck malignancies. A retrospective analysis of neck dissections performed for the management of various head and neck cancers, during 2006-2010 was carried out. Among 102 patients and 119 neck dissections, five of them were found to be having abnormalities of the cervical part of the internal carotid artery during its course in the neck and were analyzed in detail. Out of five subjects, four were males and the other was female with age ranging from 50 to 74 years. Of the five patients two had mild degree of tortuosity, two patients had moderate degree of tortuosity and another patient had severe degree of tortuosity in the course of internal carotid artery in the neck. Based on our cases, the possibilities of various vascular variations should be kept in mind while performing the neck dissection. This will help in preventing inadvertent injury to these vital structures and prevents subsequent consequences.
\end{abstract}

Keywords Internal carotid artery $\cdot$ Neck dissection . Head and neck cancer

R. B. Metgudmath · V. V. Metgudmath · V. Jainkeri Department of Otorhinolaryngology, Head and Neck Surgery, Jawaharlal Nehru Medical College, KLE University, Belgaum, Karnataka, India

A. R. Metgudmath

Department of General Medicine, Jawaharlal Nehru Medical

College, KLE University, Belgaum, Karnataka, India

R. B. Metgudmath $(\bowtie)$

95/C, Anugraha, Shanti-nagar, M.G. Road, Tilakwadi,

Belgaum 590006, Karnataka, India

e-mail: drrajmet@yahoo.co.in

\section{Introduction}

Neck dissection is one of the commonest procedures performed by head and neck surgeons. Although, the technique of neck dissection has been perfected over the last century, every surgeon encounters either a major and/or minor intra-operative or post-operative complication quite frequently than expected and a substantial knowledge of the anatomy of important structures in neck helps in avoiding many of these complications during the procedure. The carotid (internal carotid and external carotid) arteries being major source of blood supply to the head and neck region, sound knowledge about their course and variations is vital for any surgeon operating in the region of neck.

The internal carotid artery at its origin lies lateral to external carotid artery but as it ascends in the neck, the external carotid artery passes backward and laterally as shown in Fig. 1 [1]. In this article, we report variations of the internal carotid artery in the neck encountered during neck dissection near its origin, i.e., in the region of level II in the neck.

\section{Materials and Methods}

A retrospective review of the case records and intra-operative photographs of patients who underwent surgical management for head and neck tumors at the KLES. Dr. Prabhakar Kore Hospital and MRC, Belgaum, over a period of 5 years (2006-2010) was conducted and 130 patients were found to have undergone surgical excision for various head and neck tumors. Among these 102 patients, who had undergone some form of neck dissection were included in the study. Totally 119 neck dissections (17 patients had 


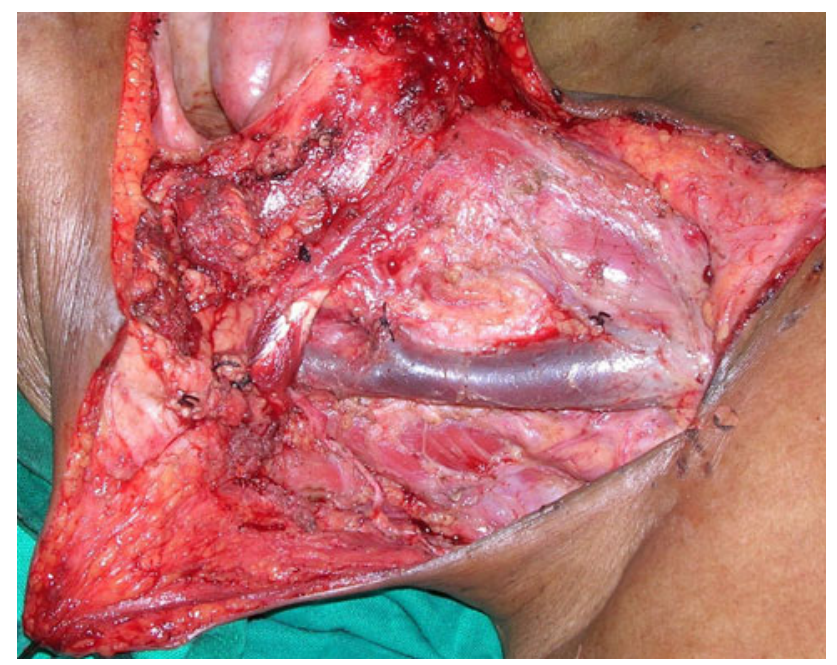

Fig. 1 Intra-operative photograph of right side neck dissection showing normal course of the internal carotid artery at level II

undergone bilateral neck dissection) were reviewed for the abnormalities of the internal carotid artery in the neck. Out of these 119 neck dissections, five were found to be having variations of the internal carotid artery in the neck and were analyzed further in detail with respect to level of variation, degree of variation and also associated intraoperative and/or post-operative complications if any.

\section{Results}

Of the 119 neck dissections and 102 patients, five patients were found to be having variations of the internal carotid artery in the neck at the region of level II (area extending from base of skull up to the hyoid bone). Among five patients, four were males and one female, with the mean age of 62 (50-74) years, corresponding with the peak age for malignancies. Among these five, two patients of carcinoma of buccal mucosa were found to be having minor degree of tortuosity (Fig. 2), a patient with carcinoma of larynx and another with carcinoma of left buccal mucosa had moderate degree of tortuosity (Fig. 3) and a patient with carcinoma of left buccal mucosa had severe degree of tortuosity (Fig. 4) of the internal carotid artery in the neck at level II near its origin. All the patients had undergone careful neck dissection and none had any form of intraoperative vascular trauma or post-operative complications in our study.

\section{Discussion}

"One, who masters the anatomy, shall master the surgery" is an age old and time tested dictum well-known to

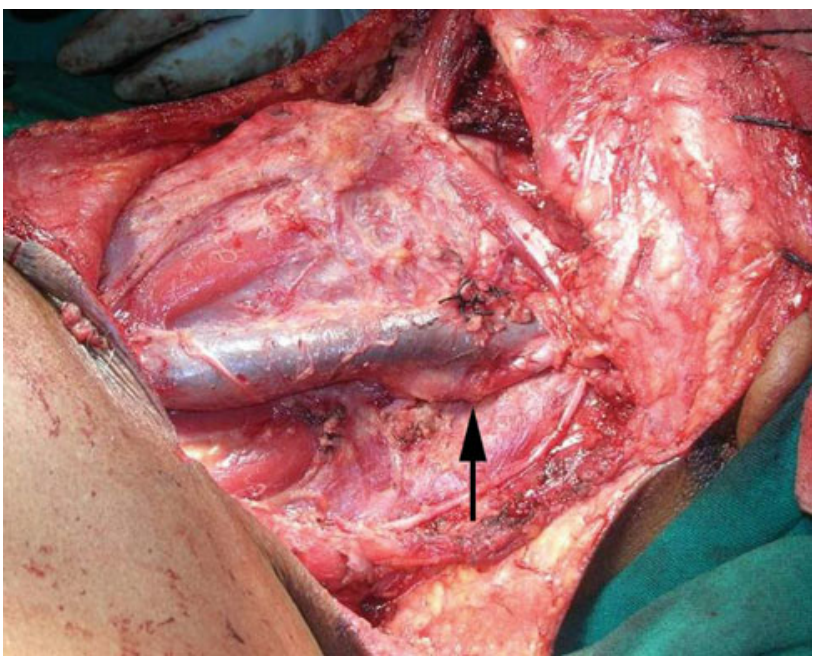

Fig. 2 Intra-operative photograph of left side neck dissection showing mild degree of variation in the course of the internal carotid artery at level II

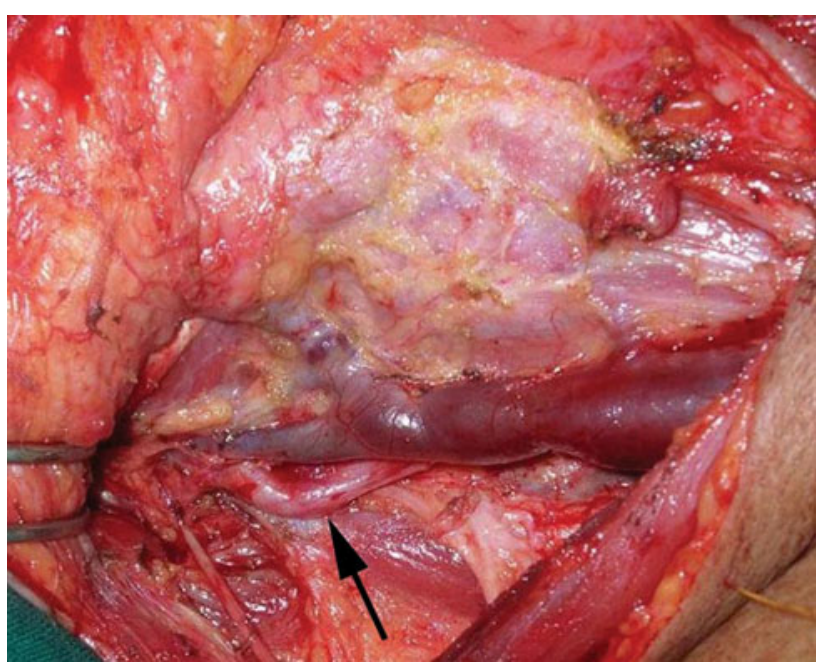

Fig. 3 Intra-operative photograph of right side neck dissection showing moderate degree of variation in the course of the internal carotid artery at level II

everyone in the milieu of surgery and it is needless to say that, same holds true in the field of head and neck surgery also. Keeping the same dictum as the basis we conducted this study.

Of the two carotid arteries in the neck, the right common carotid arises from the brachiocephalic artery, whereas left common carotid artery arises directly from the arch of aorta in the superior mediastinum. The right common carotid artery arises above the level of the sternoclavicular joint in $12 \%$ of cases [2]. The left common carotid artery has higher incidence of variation than the right and may arise with brachiocephalic artery [2]. Normally the common carotid artery divides into external and internal carotid arteries at the level of the upper border of the thyroid 


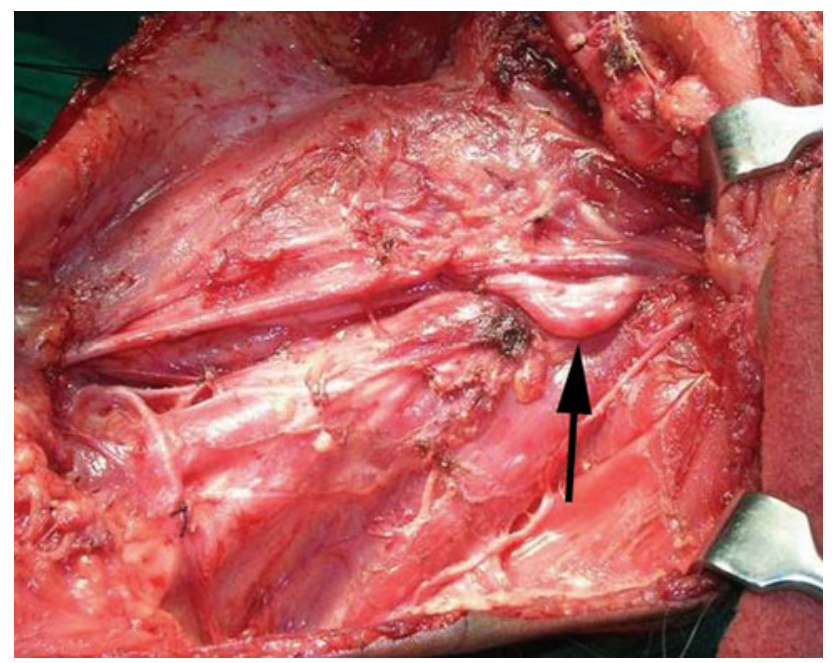

Fig. 4 Intra-operative photograph of left side neck dissection showing severe degree of variation in the course of the internal carotid artery at level II

cartilage approximately between third and fourth cervical vertebrae [1-4]. But the level of this division of the common carotid artery is highly variable and may occur as high as, the level of the hyoid bone or at a lower level alongside the larynx [2, 5]. Rarely, carotid artery is found to ascend without any division and at times it is also noticed that, common carotid artery may be replaced by separate external and internal carotid arteries which arise directly from the aorta, on one side, or bilaterally [2]. The internal carotid artery at its origin in the neck first lies lateral to external carotid artery but as it ascends in the neck, the external carotid artery passes backward and laterally (Fig. 1) [1].

In two of our cases of carcinoma of buccal mucosa, who had under gone neck dissection, the internal carotid artery had minor degree of tortuosity, i.e., part of the internal carotid artery was found to be just posterior to the internal jugular vein at level II as seen in Fig. 1.

A case of carcinoma of larynx subjected for bilateral neck dissection showed the internal carotid artery on right side had moderate degree of tortuosity, i.e., a segment of internal carotid artery was found to be completely posterior to the internal jugular vein at level II, forming a curvature as seen in Fig. 2. Another patient with carcinoma of left buccal mucosa also showed similar variation.

Lastly a case, of carcinoma of the left buccal mucosa subjected for left neck dissection revealed, the internal carotid artery of having severe degree of tortuosity, i.e., a segment of internal carotid artery was having an acute bend at level II, such that it formed a loop like curvature as seen in Fig. 3. This type of variation is more prone for vascular injury during neck dissection.

As we know the peak incidence of malignancies is present after the fifth decade which, also coincides with the peak incidence of many chronic metabolic and age related diseases like diabetes mellitus, hypertension, and atherosclerosis [6]. These diseases affect the elasticity and compliance of blood vessels either directly or indirectly which might lead to above said abnormality of the carotids [6]. In our study though none were found to be suffering from the chronic metabolic diseases, all five were aged above 50 years and hence age might have played its role in presence of these tortuosities of internal carotid artery.

During neck dissection these variations of internal carotid artery at level II should be kept in mind. If precautions are not taken the operating surgeon may inadvertently injure the vessel leading to grave complications. Nowadays at most centers virtually all patients are evaluated with some form of contrast-enhanced cross-sectional imaging, either CT or MRI, accompanied by three-dimensional coronal and sagittal reconstructions which allow the assessment of the course of major blood vessels. Hence the precise localization of the course of the carotid artery prior to surgery should be fairly standard but, in many centers of developing countries where imaging is not performed in all cases due to finical constraints, the knowledge of such variations should be kept in mind while performing neck dissection, especially when the patient is elderly or suffering from any metabolic diseases. Thus, during clearance of level II especially the junior surgeons should palpate the area posterior to internal jugular vein and feel for the pulsations. If pulsations are felt posterior to internal jugular vein at level II it gives an idea that, there is a variation in the course of internal carotid artery. Another method of avoiding injury to such variations of internal carotid artery is by performing the dissection from posterior to anterior, that is starting from level II $b$ and progressing carefully towards level II a. These methods will surly avoid the inadvertent injury which may be caused to the internal carotid artery.

In our through literature search, there was no published data of such variations or abnormalities in the course of internal carotid artery at level II as seen in our cases.

\section{Conclusion}

We herein report on various degrees of tortuosity of the proximal part of internal carotid artery. The knowledge about such vascular variations attains great importance when performing neck dissection, especially for the junior surgeons. This will in-turn help in preventing inadvertent injury to these vital structures and prevents subsequent untoward consequences.

Conflict of interest None. 


\section{References}

1. Snell RS (ed) (2004) Clinical anatomy, 7th edn. Lippincott Williams \& Wilkins, Maryland

2. Standring S (ed) (2005) Gray's anatomy: the anatomical basis of clinical practice, 39th edn. Elsevier, Philadelphia

3. Ord RA, Ward-Booth RP (1986) Anomalies of the common carotid artery: a rare complication of radical neck dissection. Br J Oral Maxillofac Surg 24:405-409
4. Moore KL, Dally AF (eds) (1999) Clinically oriented anatomy, 4th edn. Lippincott Williams \& Wilkins, Maryland

5. Putz R, Pabst R (eds) (2006) Sobotta atlas of human anatomy, 14th edn. Elsevier, Munchen

6. McVeigh GE (1996) Arterial compliance in hypertension and diabetes mellitus. Am J Nephrol 16(3):217-222 